\title{
REV-ERB $\alpha$ Activates C/EBP Homologous Protein to Control Small Heterodimer Partner-Mediated Oscillation of Alcoholic Fatty Liver
}

\author{
Zhihong Yang, ${ }^{* \dagger}$ Hiroyuki Tsuchiya, ${ }^{\ddagger}$ Yuxia Zhang, ${ }^{\S}$ Sangmin Lee, ${ }^{*}$ Chune Liu, ${ }^{*}$ Yi Huang, ${ }^{* \llbracket}$ Gymar M. Vargas, ${ }^{*}$ and Li Wang ${ }^{* \dagger \Phi \|}$
}

\begin{abstract}
From the Department of Physiology and Neurobiology, and the Institute for Systems Genomics, * University of Connecticut, Storrs, Connecticut; the Veterans Affairs Connecticut Healthcare System, ${ }^{\dagger}$ West Haven, Connecticut; the Graduate School of Pharmaceutical Sciences, ${ }^{\ddagger}$ Osaka University, Osaka, Japan; the Department of Pharmacology, Toxicology and Therapeutics, ${ }^{\S}$ University of Kansas Medical Center, Kansas City, Kansas; the School of Pharmaceutical Sciences, "Wenzhou Medical University, Wenzhou, Zhejiang, China; and the Section of Digestive Diseases, "Department of Internal Medicine, Yale University, New Haven, Connecticut
\end{abstract}

\author{
Accepted for publication \\ July 11, 2016. \\ Address correspondence to \\ Li Wang, Ph.D., University of \\ Connecticut, $75 \mathrm{~N}$ Eagleville \\ Rd, Unit 3156, Storrs, CT \\ 06269. E-mail: li.wang@ \\ uconn.edu.
}

\begin{abstract}
The small heterodimer partner (SHP) nuclear receptor is an important regulator of nonalcoholic fatty liver disease. However, little is known about the role of SHP in alcoholic fatty liver. In this study, we used a modified chronic ethanol-binge model to examine cyclic alterations of lipid metabolism in wildtype (WT) and $\mathrm{Shp}^{-1-}$ mice over a 24 -hour period after binge. The serum and hepatic lipid profiles, as well as the expression of lipid synthesis genes and markers of endoplasmic reticulum stress, exhibited distinct variations in WT and $S h p^{-/-}$mice in response to ethanol diet plus ethanol binge (ED+E) and control diet plus maltose binge. ED+E induced steatosis in WT mice, which correlated with a marked upregulation of activating transcription factor 4 protein (ATF4) but down-regulation of C/EBP homologous protein (CHOP) and sterol regulatory element-binding transcription factor 1c protein (SREBP-1c). On the contrary, the control diet plus maltose binge caused lipid accumulation in $\mathrm{Sh}^{-/-}$mice, which was accompanied by a sharp elevation of CHOP, SREBP-1C, and REV-ERB $\alpha$ proteins but a diminished ATF4. REV-ERB $\alpha$ activated CHOP promoter activity and gene transcription, which were inhibited by SHP. Knockdown Rev-Erb $\alpha$ in $\mathrm{Shp}^{-/-}$mice prevented steatosis induced by ED+E. Our study revealed a critical role of SHP and REV-ERB $\alpha$ in controlling rhythmic CHOP expression in alcoholic fatty liver. (Am J Pathol 2016, 186: 2909-2920; http://dx.doi.org/10.1016/j.ajpath.2016.07.014)
\end{abstract}

Liver is a major organ to detoxify alcohol and thus is prone to be damaged by alcohol abuse. Research has been well established that excessive consumption of alcohol results in the development and progression of chronic liver disease, including liver steatosis, steatohepatitis, cirrhosis, and hepatocellular carcinoma. ${ }^{1,2}$ Although mechanisms that lead to these pathologies have been studied extensively, the development of an effective therapy for alcoholic liver disease (ALD) remains challenging.

The endoplasmic reticulum (ER) stress response is a fundamental reaction seen even in healthy liver and has been noted to increase after alcohol intake. ${ }^{3}$ The ER stress response, at steady state, plays a protective role against such toxic factors by removing misfolded and/or unfolded proteins from the ER. However, when the stress becomes persistent and strong, the response switches to the induction of liver steatosis, insulin resistance, and apoptosis. ${ }^{4}$ Various factors have been identified as causes for alcohol-induced ER stress response, such as oxidative stress, acetoaldehyde, free fatty acid, insulin resistance, disturbance of calcium homeostasis, and disruption of one carbon metabolism, all of which are known to impair ER functions. 3,5

Small heterodimer partner (SHP; NR0B2) is an atypical orphan nuclear receptor mainly expressed in the liver. ${ }^{6,7}$

Supported by NIH R01DK104656, R01DK080440, R01ES025909, R21AA022482, R21AA024935, VA Merit Award 1I01BX002634, and P30 DK34989 (Yale Liver Center) (L.W.); and National Natural Scientific Foundation of China grant 81572443 (Y.H.).

Disclosures: None declared. 
Unlike many other nuclear receptors, SHP lacks DNAbinding domain and inhibits transcriptional activity of other transcription factors. ${ }^{8}$ Our previous studies revealed a central role of SHP in liver metabolism, including bile acid, lipid, and one carbon metabolism. ${ }^{5,9-11}$ Specifically, SHP inhibited very-low-density lipoprotein secretion by repressing microsomal triglyceride transfer protein $^{11}$ expression and played a key role to control diurnal regulation of plasma triglyceride by the circadian locomotor output cycles kaput gene. ${ }^{12}$ Consequently, $\mathrm{Shp}^{-/-}$mice were resistant to highfat diet induced fatty liver. ${ }^{13}$ We recently demonstrated that SHP was an essential component of the liver circadian clock machinery through interaction with RAR-related orphan receptor $(\mathrm{ROR} \gamma)$ and nuclear receptor subfamily 1 group D1 (REV-ERB $\alpha$ ) to regulate neuronal PAS domaincontaining protein $2-$ mediated hepatic lipid metabolism. ${ }^{14}$ Neuronal PAS domain-containing protein 2 was induced in $S h p^{-/-}$mice and knockdown neuronal PAS domaincontaining protein 2 attenuated very-low-density lipoprotein production and sensitized $\mathrm{Sh}^{-1-}$ mice to develop steatosis. ${ }^{14}$ Despite these previous studies that elucidated a critical role of SHP in nonalcoholic fatty liver disease (NAFLD), the regulatory function of SHP in alcoholic fatty liver (AFL) remained elusive.

Although the role of the ER stress response has been established in alcoholic fatty liver diseases, the importance of circadian clock in the ER stress response in ALD, and the role of diet although intriguing, remains largely unexplored. The objective is to understand the function of SHP in integrating the liver circadian clock with alcohol-induced ER stress response and steatosis. The hypothesis is that genes in ER stress and lipid metabolism are under the control of liver clock that is modulated by alcohol and SHP. In the present study, we elucidated a critical cross talk between SHP and REV-ERB $\alpha$ in the regulation of alcohol-induced steatosis involving $\mathrm{C} / \mathrm{EBP}$ homologous protein (CHOP). Our study provides novel insight into SHP and REV-ERB $\alpha$ control of ER stress signaling associated with the development of AFL.

\section{Materials and Methods}

\section{Cell Culture and Animals}

Mouse hepatoma cell line Hepa 1-6 (ATCC, Manassas, VA; CRL-1830) was maintained in Dulbecco's modified Eagle's medium (11965-118; Invitrogen, Carlsbad, CA) with $100 \mathrm{U}$ of penicillin G-streptomycin sulfate/mL and $10 \%$ heatinactivated fetal bovine serum (10437-028; Gibco, Waltham, MA). C57BL/6J mice were purchased from Jackson Laboratory (Bar Harbor, ME). Shp ${ }^{-1-}$ on C57BL/6J background was described previously. ${ }^{10,14}$ Mice were fed a standard rodent chow with free access to water and maintained in a 12-hour light/dark cycle (light on 6 AM to 6 PM), temperaturecontrolled $\left(23^{\circ} \mathrm{C}\right)$, and virus-free facility. Experiments were performed on male mice at the age of 8 to 12 weeks unless stated otherwise ( $n=5$ per group). For ethanol feeding, the
Gao-binge model was used with slight modification. ${ }^{1,5,15}$ In brief, the mice were acclimated to a control liquid diet (F1259SP; Bio-Serv, Flemington, NJ) for 5 days and followed by control liquid diet or 5\% Lieber-DeCarli ethanol liquid diet (F1258SP; Bio-Serv) for 10 days. On day 11 at 9 AM the mice were challenged by oral gavage of a single dose of maltose dextrin $(9 \mathrm{~g} / \mathrm{kg}$ of body weight) or ethanol $(5 \mathrm{~g} / \mathrm{kg}$ of body weight) solution. Nine hours after the binge, blood samples and liver tissues were collected every 6 hours over a 24-hour light/ dark cycle at Zeitgeber time (ZT) 12, ZT18, ZT0, and ZT6. All experiments were performed in accordance with relevant guidelines and regulations approved by the Institutional Animal Care and Use Committee at the University of Connecticut (Storrs).

\section{Plasmids and Viruses}

The mouse Chop (Gene ID 13198) promoter luciferase reporter (pGL3-mChop-pro) and its mutation construct (pGL3mChop-pro-mut) were engineered in our laboratory. In brief, the mouse Chop promoter was amplified by Takara LA Taq Polymerase (RR002A; Takara Bio, Shiga, Japan) using wildtype (WT) mice genomic DNA as a template and cloned into pGL3-basic (E1751; Promega, Madison, WI). pGL3-mChoppro-mut was generated by a QuikChange Site-Directed Mutagenesis Kit (200521; Stratagene, San Diego, CA). The integrity of the recombinant plasmids was verified by sequence analysis. Two shRNAs for knockdown mouse RevErb $\alpha$ (TRCN0000027094 and TRCN0000027070) and one shRNA control plasmid in pLKO.1 vector (SHC002) were purchased from Sigma (St. Louis, MO). The sequences of the shRNAs were provided in Table 1. For adeno-associated virus-8 (AAV8)-mediated shRNA knockdown of RevErb $\alpha$, U6-Rev-Erb $\alpha$-shRNA-2 cassette was cloned into an AAV8 vector and virus particles were produced by Vector BioLabs (Malvern, PA). Mice were injected via tail vein with purified AAV8 virus at $1 \times 10^{11}$ virus particles per mouse once a week for 2 weeks and subjected to Gao-binge model. Serum, liver, and brain tissues were collected at 21 hours (ZT0) after oral gavage. The in vivo adenoviral transduction of SHP (adSHP) and its control green fluorescent protein (adGFP) into $S h p^{-/-}$mice has been described previously. ${ }^{14,16}$ The $S h p^{-1-} /$ adGFP and $S h p^{-1-} /$ adSHP mice were fed with standard chow diet and the liver tissues were collected every 4 hours over a 24-hour light/dark cycle at ZT2, ZT6, ZT10, ZT14, ZT18, and ZT22.

\section{In Vivo Plasmid Transfection}

The expression plasmids pcDNA3 and pcDNA3-Flag-RevErb $\alpha$ were described previously. ${ }^{14}$ shRNAs for mouse Atf4 (TRCN0000301646) were purchased from Sigma. In vivo plasmid transfection was performed using TurboFect in vivo Transfection Reagent (R0541; Thermo Scientific, Waltham, MA). Plasmids $(50 \mu \mathrm{g})$ were diluted in $400 \mu \mathrm{L} \mathrm{5 \%}$ glucose solution, mixed with $6 \mu \mathrm{L}$ of in vivo transfection reagent, and 
Table 1 Primer Sequences

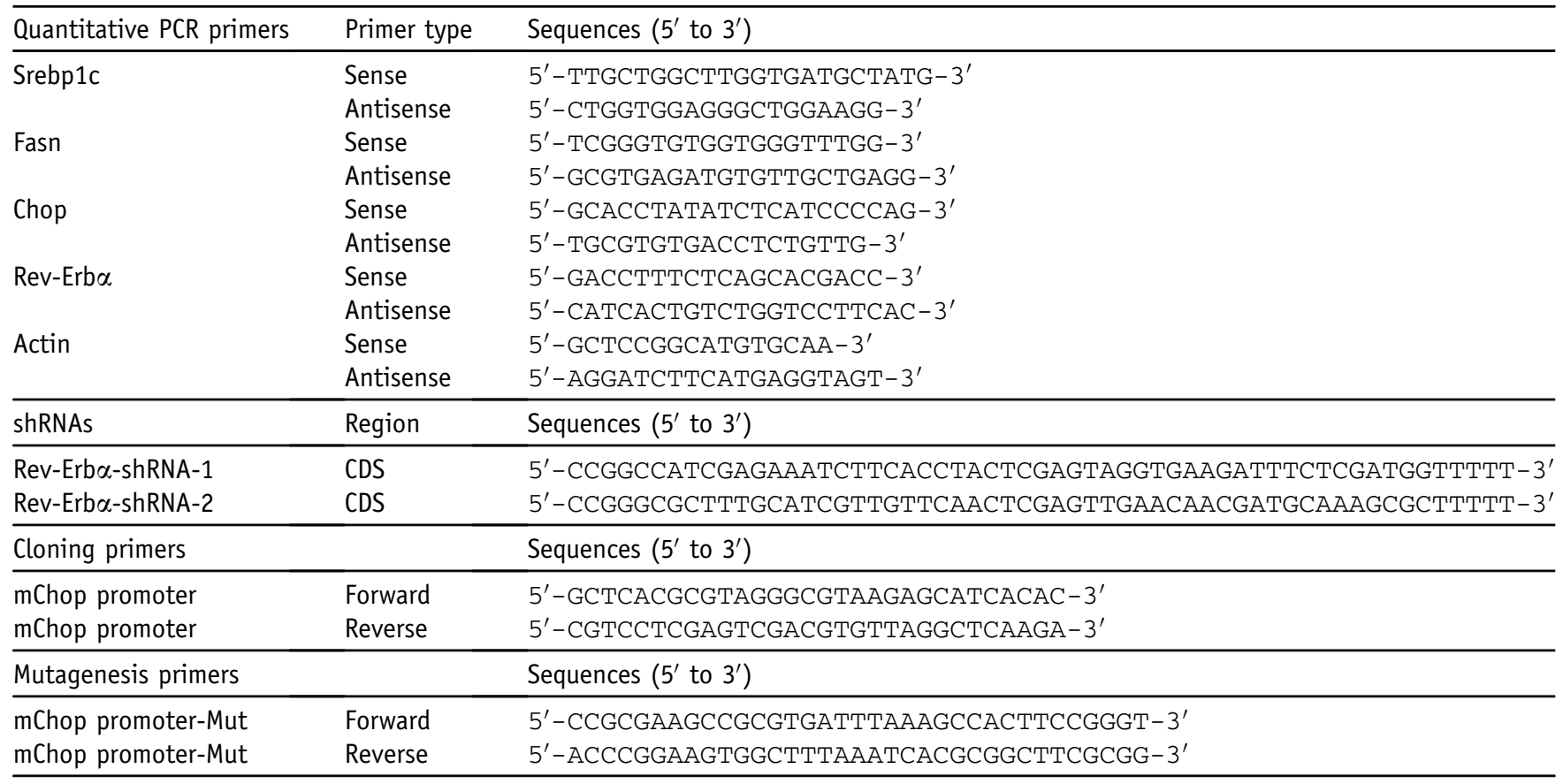

CDS, coding sequence; Chop, C/EBP homologous protein; Fasn, fatty acid synthase; mChop, mouse Chop; Mut, mutation; Srebp1c, sterol regulatory elementbinding transcription factor $1 \mathrm{c}$.

incubated for 20 minutes before injected to WT mice through tail vein. Serum and liver tissues were collected at day 4 after injection. For shAtf4 knockdown, pLKO-shAtf4 or control vector $\mathrm{PLKO}$ plasmids were injected at day 1 and day 5 during the ethanol feeding period. After ethanol binge, serum and liver tissues were collected at ZT12.

\section{Measurements of TG and ALT/AST Levels}

Serum and liver triglyceride (TG) levels were determined as described previously. ${ }^{14}$ Briefly, lipid was extracted from $200 \mathrm{mg}$ liver tissue into $600 \mu \mathrm{L}$ chloroform-methanol mix (2:1) and redissolved in $500 \mu \mathrm{L}$ phosphate-buffered saline (pH 7.4) with 5\% Triton X-100. Serum TG or dissolved liver TG was measured using Pointe Scientific Triglycerides Liquid Reagents (Thermo Fisher Scientific; 23-666-410), according to the manufacturer's instructions. Each sample was run in duplicate. Serum levels of aspartate aminotransferase (AST) and alanine aminotransferase (ALT) were determined by Infinity AST (glutamate oxalacetate transaminase) and ALT (glutamate pyruvate transaminase) reagents (Thermo Fisher Scientific; TR70121 and TR71121).

\section{Histological Analysis of Liver Sections}

Liver tissue fixing with formalin was described previously. ${ }^{10}$ Paraffin sections were cut at thickness of $4 \mu \mathrm{m}$ and subjected to hematoxylin and eosin staining and periodic acid-Schiff staining. The images were captured using Olympus BX41 microscope (Olympus, Lake Success, NY). The random fields from each liver section were captured and analyzed.

\section{RNA Isolation and Quantitative PCR}

Total RNA was isolated using TRIzol Reagent (15596-018; Invitrogen), and cDNA synthesis was performed with HighCapacity cDNA Reverse Transcription Kit (4368813; Applied Biosystems, Foster City, CA). The quantitative PCR was performed using the iTaq Universal SYBR Green Supermix (1725124; Bio-Rad, Hercules, CA) with primers shown in Table 1. The relative ratio of specific genes to internal control $\beta$-actin for each sample was then calculated.

\section{Western Blots}

Hepal cells or liver tissues were lysed as described previously. ${ }^{8}$ The concentration of protein was measured using Pierce BCA Protein Assay Kit (Thermo Fisher; 23227). Cell lysates $(30 \mu \mathrm{g})$ were resolved by SDS-PAGE and transferred to nitrocellulose membranes by standard procedures. Antibody binding was visualized with SuperSignal West Pico Chemiluminescent Substrate (Thermo Fisher Scientific; 34080), according to the manufacturer's protocol. Equal loading of protein was verified with $\beta$-actin, glyceraldehyde-3-phosphate dehydrogenase, or tubulin. Quantitative analysis of the band intensity was performed by ImageJ software version $1.50(\mathrm{NIH}$, Bethesda, MD; http://imagej. nih.gov/ij).

Western blots of CHOP and activating transcription factor 4 (ATF4) proteins were performed in $97 \mathrm{H}$ cells treated with tunicamycin (ER stress inducer). MHC97H cells were fasted for 24 hours before treatment with tunicamycin $(1 \mathrm{ng} / \mathrm{mL})$ for 24 hours. As MHC97H cells have low basal levels of 
ATF4 and CHOP, they were chosen to determine their induction by tunicamycin.

The following antibodies were used for primary antibody incubation: $\beta$-actin (sc-47778; Santa Cruz Biotechnology, Dallas, TX), sterol regulatory element-binding transcription factor 1c (SREBP-1c; MA5-11685; Thermo Fisher Scientific), acetyl-CoA carboxylase (sc-30212; Santa Cruz Biotechnology), fatty acid synthase (sc-20140; Santa Cruz Biotechnology), PRKR-like endoplasmic reticulum kinase (3192; Cell Signaling Technology, Danvers, MA), inositolrequiring enzyme $\alpha$ (3294; Cell Signaling Technology), ATF6 (ab122897; Abcam, Cambridge, UK), total eukaryotic translation initiation factor $2 \propto(5324 \mathrm{p}$; Cell Signaling Technology), phosphorylated eukaryotic translation initiation factor $2 \alpha$ (3398p; Cell Signaling Technology), ATF4 (11815s; Cell Signaling Technology), CHOP (2895p; Cell Signaling Technology), p-AKT (9271; Cell Signaling Technology), REV-ERB $\alpha$ (SAB2101632; Sigma), and FLAG (F3165-1MG; Sigma).

\section{Knockdown Experiment}

\section{REV-ERB $\alpha$}

After acclimatization for 5 days, mice were given control or ethanol-containing Lieber-DeCarli liquid diets for 10 days, followed by oral gavage of maltose (control) or ethanol solutions at 9 AM on day 10. WT mice were injected with AAV8-driven control or shRNA for Rev-Erb $\alpha$ through the tail vain. Two weeks later, the mice were injected again and subject to the Gao-binge protocol after 3 days. Livers and sera were collected at ZT0.

\section{ATF4}

WT mice were injected with pLKO or pLKO-sh-Atf4 plasmids $(50 \mu \mathrm{g}$ per mice) on days 1 and 5 of ethanol feeding using the TurboFect in vivo Transfection Reagent following the manufacturer's instructions. On day 11, mice were orally gavaged with maltose or ethanol. Livers and sera were collected at ZT12.

\section{Luciferase Assay}

Hepa1-6 cells in 24-well plates were cotransfected with mouse Chop promoter (pGL3-mChop-pro), mouse Chop promoter mutant (pGL3-mChop-pro-mut), pcDNA3-FlagShp, pcDNA3-Flag-Rev-Erb $\alpha$, or pcDNA3-ROR $\alpha$, as indicated in figure legends using transfection reagent X-tremeGENE HP (06366236001; Roche, Basel, Switzerland). ${ }^{17}$ In the ethanol group, 48 hours after transfection, cells were cultured in fetal bovine serum-free Dulbecco's modified Eagle's medium for 3 hours and followed by $400 \mathrm{mmol} / \mathrm{L}$ ethanol treatment for an additional 6 hours. Luciferase and renilla activities were determined by Dual-Luciferase Reporter Assay Systems (Promega; E1960). Luciferase activity was normalized by renilla activity expressed from cotransfected pSV-renilla. Each data point was the average of triplicate assays and repeated three times.

\section{Statistical Analysis}

All of the experiments were performed in triplicate and repeated at least three times. The data are presented as the means \pm SEM. Statistical analysis was performed using the Student's $t$-test for unpaired data to compare the values between the two groups. $P<0.05$ was considered statistically significant.

\section{Results}

\section{Shp ${ }^{-1-}$ Mice Developed Different Types of Steatosis in Response to $\mathrm{ED}+\mathrm{E}$ and $\mathrm{CD}+\mathrm{M}$}

The recently developed Gao-binge model ${ }^{15}$ represents an acute model of alcohol-induced liver injury. It is easy to perform, thus has been widely used by other research groups. ${ }^{18,19}$ One observation was that the changes in gene expression and serum enzymes were transient after binge. ${ }^{15}$ To have a better understanding of the phenotypic changes, we collected samples over a 24-hour light/dark cycle (Supplemental Figure S1). ${ }^{15}$ To establish the physiological relevance of SHP in AFL, we first examined Shp expression in WT mice after ethanol diet plus ethanol binge $(E D+E)$. Interestingly, the expression of Shp in the control diet plus maltose binge $(\mathrm{CD}+\mathrm{M})$ group appeared rhythmic, which was low during the night and high during the day (Figure 1A). Shp mRNA was markedly reduced 9 hours after ethanol binge compared with maltose binge (ZT12) (Figure 1A), which was gradually recovered to the same level as in the $\mathrm{CD}+\mathrm{M}$ group after 24 hours. We did not detect Shp mRNA in $S h p^{-/-}$mice (data not shown). The quantitative PCR results were in agreement with the RNA-sequencing data (Supplemental Figure S2A).

Liver and serum TG levels exhibited distinct variations in both WT and $S h p^{-1-}$ mice after the maltose or ethanol binge over a 24-hour light/dark cycle. The liver TG content in WT mice was increased 9 hours after ethanol binge (ZT12), and was decreased to the normal level after 24 hours (ZT6), as compared to the $\mathrm{CD}+\mathrm{M}$ (Figure 1B). Remarkably, the liver TG content in $S h p^{-/}$mice showed a striking induction and oscillation in $\mathrm{CD}+\mathrm{M}$, which was decreased in ED+E. Serum TG showed dissimilar rhythmic changes (Figure 1C). ED + E-induced serum TG reached the highest level at ZT0 in WT mice, which remained in a constant high level from ZT12 to ZT6 in $S h p^{-1-}$ mice. The decreased liver TG at several time points in WT and $\mathrm{Shp}^{--}$mice correlated with the increased serum TG, suggesting a dynamic oscillation in hepatic lipid secretion during the 24-hour light/dark cycle. The induction in serum TG in $S_{h} p^{-1-} \mathrm{CD}+\mathrm{M}$ mice also 
A

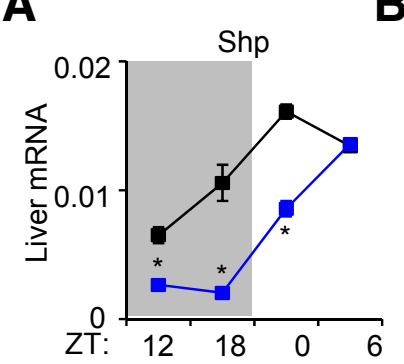

B

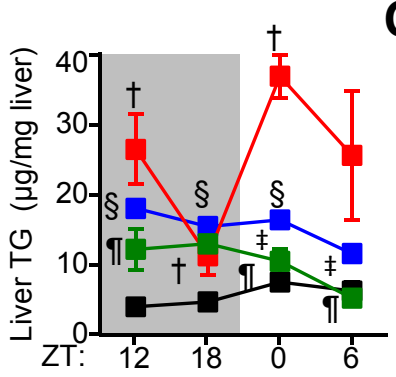

C

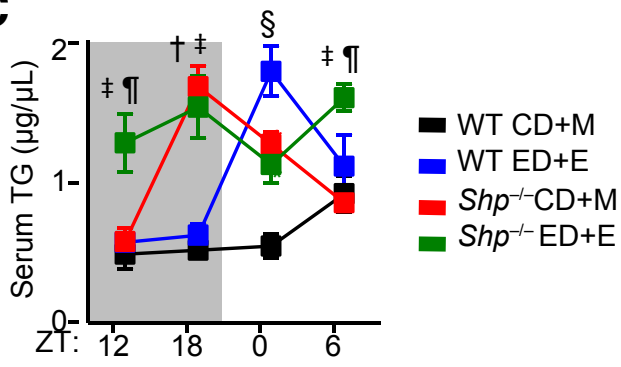

D

WT
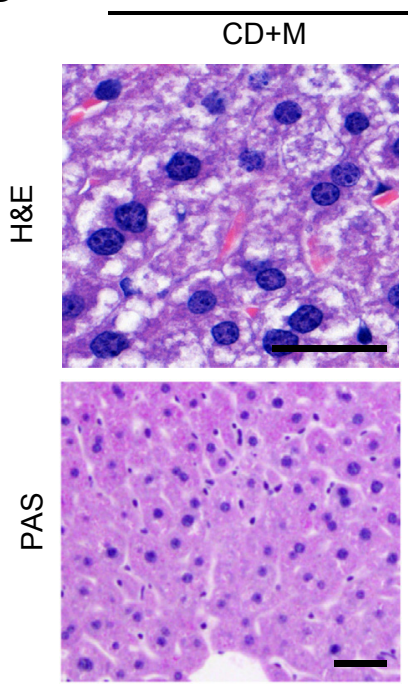

E

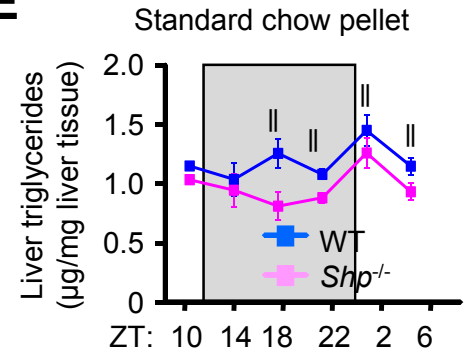

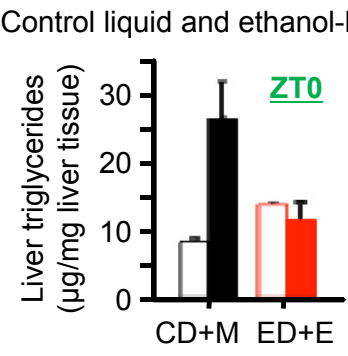

Shp $p^{-1-}$
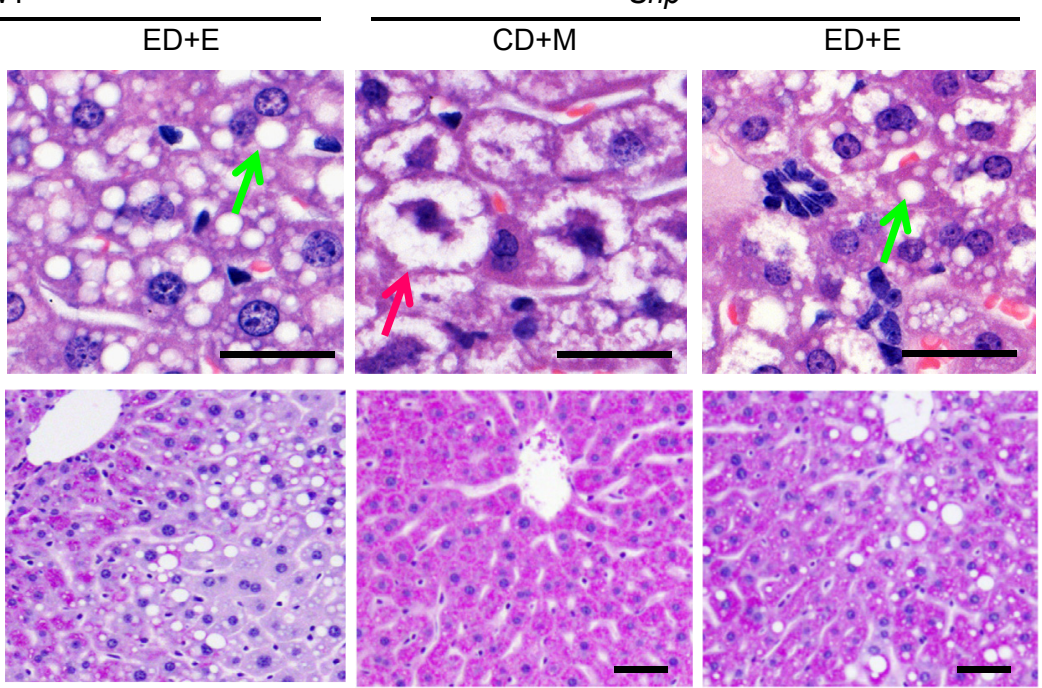

Figure 1 Shp deficiency altered maltose- and ethanol-mediated alteration of lipid metabolism. A: Quantitative PCR of mRNA expression of Shp in WT mice fed control diet + maltose binge $(C D+M$; black) or ethanol diet + ethanol binge (ED+E; blue). Each lane represents triplicate assays from equal amounts of mRNA pooled from five individual mice. The Gao-binge model was used. The samples were collected over a 12-hour/12-hour light/dark cycle 9 hours after the binge at Zeitgeber time (ZT) 12, 18, 0, and 6. Gray shading indicates dark cycle; white indicates light cycle. B and C: Liver (B) and serum (C) triglyceride (TG) contents in WT (black and blue) and $S h p^{-1-}$ (red and green) mice fed CD+M (black and red) or ED+E (blue and green). D: Representative images of hematoxylin and eosin (H\&E; top row) and periodic acid-Schiff (PAS; bottom row) staining of liver sections from WT and Shp ${ }^{-/-}$mice fed CD+M or ED+E at ZT0. We use different magnification to convey the results more clearly. Green arrows indicate macrosteatosis; red arrow, microsteatosis. E: Left panel: Liver TG contents in WT (blue) and Shp ${ }^{-/-}$(magenta) mice fed a standard chow pellet diet. The livers were collected over a 12-hour/12-hour light/dark cycle at ZT10, 14, 18, 22, 2, and 6. Middle panel: Liver TG contents in WT (blank) and Shp ${ }^{-/}$(filled) mice fed CD+M (black) or ED+E (red) at ZT0. Right panel: Liver TG contents in WT (blue) and Shp ${ }^{-/}$(magenta) mice fed a standard chow pellet diet at ZT6. Data are expressed as means \pm SD (A-C and E). $n=5$ per time point per group. ${ }^{\star} P<0.05 \mathrm{WT} \mathrm{CD}+\mathrm{M}$ versus WT ED+E; ${ }^{\dagger} P<0.05 \mathrm{WT} \mathrm{CD}+\mathrm{M}$ versus $S h p^{-/-} \mathrm{CD}+\mathrm{M} ;{ }^{\ddagger} P<0.05 \mathrm{WT}$ ED+E versus $S h p{ }^{-/-} \mathrm{ED}+\mathrm{E} ;{ }^{\S} P<0.05 \mathrm{WT} \mathrm{CD}+\mathrm{M}$ versus WT ED+E; ${ }^{\top} P<0.05$ Shp $^{-/-} \mathrm{CD}+\mathrm{M}$ versus Shp ${ }^{-/-} \mathrm{ED}+\mathrm{E} ; \| P<0.05$ WT chow versus Shp ${ }^{-/-}$chow (E). Scale bar $=20 \mu \mathrm{m}(\mathbf{D}) .0$ riginal magnifications: $\times 40$ (D, top row); $\times 20$ (D, bottom row).

correlated with high serum ALT and AST levels, as shown in our recent publication. ${ }^{5}$

Hematoxylin and eosin staining revealed steatosis in WT-ED+E and $S_{h}{ }^{-/-}$-ED+E groups (Figure 1D). Interestingly, we observed a variant form of hepatic fat accumulation in $\mathrm{Shp}^{-/-}-\mathrm{CD}+\mathrm{M}$, which was characterized by small intracytoplasmic fat vacuoles accumulated in the cell, similar to microvesicular steatosis. Furthermore, periodic acid-Schiff staining revealed increased glycogen storage in $\mathrm{Shp}^{-1-}$. $\mathrm{CD}+\mathrm{M}$, and to a lesser extent in $S h p^{-/-}$-ED+E (Figure 1D).

Previous studies have shown that Shp deficiency prevented the development of steatosis under normal chow and 
high-fat diet. ${ }^{11,13,14,20}$ Because liver lipid metabolism is under the control of circadian clock, we detected liver TG levels in WT and $S h p^{-1-}$ mice fed a standard chow over a 24-hour light/dark cycle. Overall, the liver TG showed a diurnal rhythm and was diminished in $S h p^{-1-}$ versus WT mice (Figure 1E). In contrast, we observed an induction of liver TG in $S h p^{-/-}$fed with CD+M. Therefore, $S h p^{-/-}$ mice had reduction of hepatic TG contents under regular chow diet but induction of liver TG in CD+M (Figure 1E). Taken together, the results suggest that $\mathrm{ED}+\mathrm{E}$ transiently induces hepatic steatosis in WT mice and $\mathrm{Shp}^{-/-}$mice.

Ethanol Diet Plus Ethanol Binge Inactivated SREBP-1C Pathway in WT Mice, whereas Control Diet Plus Maltose Binge Activated SREBP-1c Pathway in Shp ${ }^{-/-}$Mice

SREBP-1c is a key regulator of lipid metabolism ${ }^{21}$ by activating fatty acid synthase and acetyl-CoA carboxylase to induce lipid accumulation. ${ }^{22}$ Surprisingly, both the precursor (p) and processed nuclear (n) forms of SREBP-1c proteins were decreased in WT-ED+E versus $\mathrm{WT}-\mathrm{CD}+\mathrm{M}$; on the other hand, they were highly increased in $\mathrm{Shp}^{-1-}$. $\mathrm{CD}+\mathrm{M}$ versus WT-CD $+\mathrm{M}$, and decreased in $\mathrm{Shp}^{-/-}$. $\mathrm{ED}+\mathrm{E}$ versus $\mathrm{Shp}^{-/-}-\mathrm{CD}+\mathrm{M}$ (Figure $2 \mathrm{~A}$ ). Fatty acid synthase and acetyl-CoA carboxylase proteins showed similar changes that correlated with SREBP-1c protein. The changes at SREBP-1c and fatty acid synthase proteins also occurred at the mRNA level, as determined by quantitative PCR (Figure 2B) and RNA-sequencing (Supplemental Figure S2B). The activation of SREBP-1c pathway by maltose binge may contribute to the accumulation of liver $\mathrm{TG}$ in $S h p^{-/-}-\mathrm{CD}+\mathrm{M}$, whereas the inactivation of this pathway by ethanol binge does not explain the increased liver TG in WT-ED+E. The results suggest that alternative pathways may contribute to the observed phenotype.

\section{Ethanol Diet Plus Ethanol Binge Induced ATF4 Protein in WT Mice, whereas Control Diet Plus Maltose Binge Activated CHOP and REV-ERB $\alpha$ Proteins in Shp ${ }^{-/-}$Mice}

The ER stress signaling pathways consist of three arms, inositol-requiring enzyme $1 \alpha$, PRKR-like endoplasmic reticulum kinase, and ATF6 (Supplemental Figure S3), which were often dysregulated in AFL., ${ }^{3,23}$ To identify proteins that were regulated by SHP, we analyzed major ER stress markers and observed interesting expression profiles in WT and $S h p^{-1-}$ mice fed with $\mathrm{CD}+\mathrm{M}$ and $\mathrm{ED}+\mathrm{E}$. Overall, most proteins showed modest to strong cyclic variations over a 24-hour dark (ZT12 to ZT18)/light (ZT0 to ZT6) cycle in WT-CD $+\mathrm{M}$, which were disrupted to various extents in WT-ED+E, $S h p^{-/-} \mathrm{CD}+\mathrm{M}$, and $\mathrm{Sh}^{-{ }^{-1}}{ }^{-\mathrm{ED}+\mathrm{E}}$ (Figure 3A). Specifically, ATF6 and inositol-requiring enzyme $1 \propto$ proteins were moderately increased, whereas the PRKR-like endoplasmic reticulum kinase protein was modestly down-regulated in $\mathrm{Sh}^{-1-}$ versus WT. Furthermore, total eukaryotic translation initiation factor $2 \alpha$ was induced in $S h p^{-1-}$-ED+E versus WT-ED+E, whereas phosphorylated eukaryotic translation initiation factor $2 \alpha$ was noticeably induced in WT-ED+E relative to other groups. The ATF4 protein showed the most striking
A

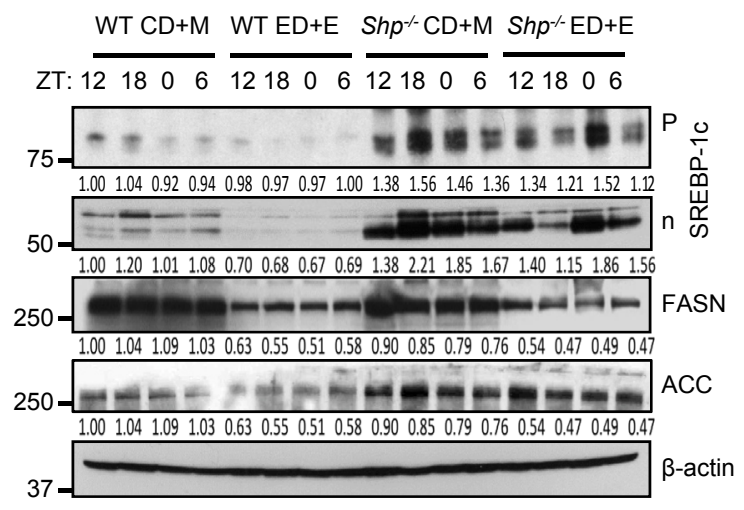

B
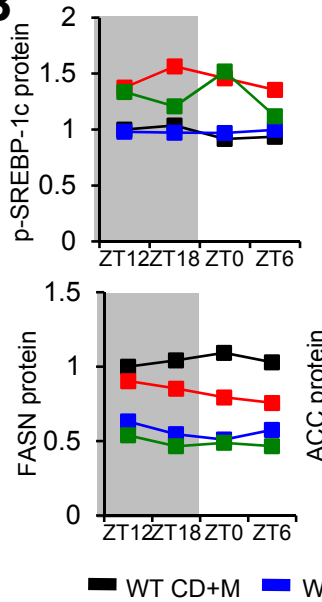
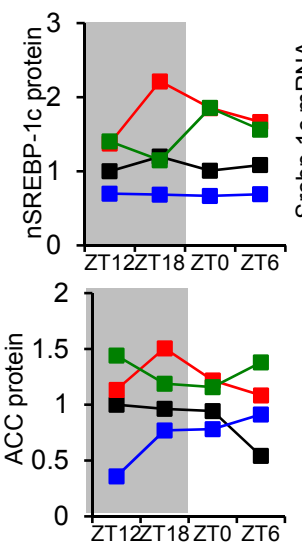
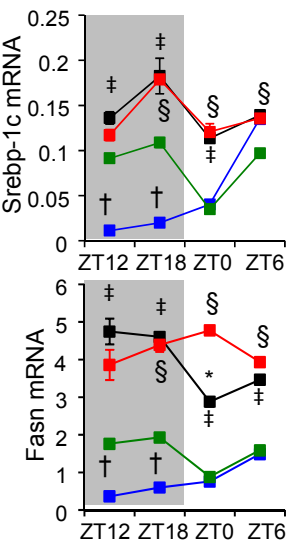

WT CD+M WT ED+E $=S h p^{-/-} \mathrm{CD}+\mathrm{M}=S h p^{-1-} \mathrm{ED}+\mathrm{E}$

Figure 2 Shp deficiency altered hepatic lipogenic markers in response to maltose and ethanol feeding. A: Western blot of hepatic lipogenic proteins in WT and $\mathrm{Shp}^{-1-}$ mice fed control diet + maltose binge $(\mathrm{CD}+\mathrm{M})$ or ethanol diet + ethanol binge $(\mathrm{ED}+\mathrm{E})$. Quantification of the intensity of each band was provided under each line. Each lane represents equal amounts of protein pooled from five individual mice. Results are quantified in panel B. B: Quantitative PCR of mRNA expression of hepatic lipogenic genes in WT (black and blue) and Shp ${ }^{-1-}$ (red and green) mice fed CD+M (black and red) or ED+E (blue and green). Each lane represents triplicate assays from equal amounts of mRNA pooled from five individual mice. Gray shading indicates dark cycle; white indicates light cycle. Data are expressed as means (left and middle column) or means $\pm \mathrm{SD}$ (right column). ${ }^{*} P<0.05 \mathrm{WT}$ CD+M versus $S h p^{-1-} \mathrm{CD}+\mathrm{M} ;{ }^{\dagger} P<0.05 \mathrm{WT} E \mathrm{ED}+\mathrm{E}$ versus $S h p^{-\prime}$ - ED+E; ${ }^{\ddagger} P<0.05$ WT CD + M versus WT ED $+\mathrm{E} ;{ }^{\S} P<0.05 \mathrm{Sh}^{-/-} \mathrm{CD}+\mathrm{M}$ versus Shp ${ }^{-/-}$ED $+\mathrm{E}$. ACC, acetyl-CoA carboxylase; FASN, fatty acid synthase; SREBP$1 \mathrm{c}$, sterol regulatory element-binding transcription factor $1 \mathrm{c}$. 


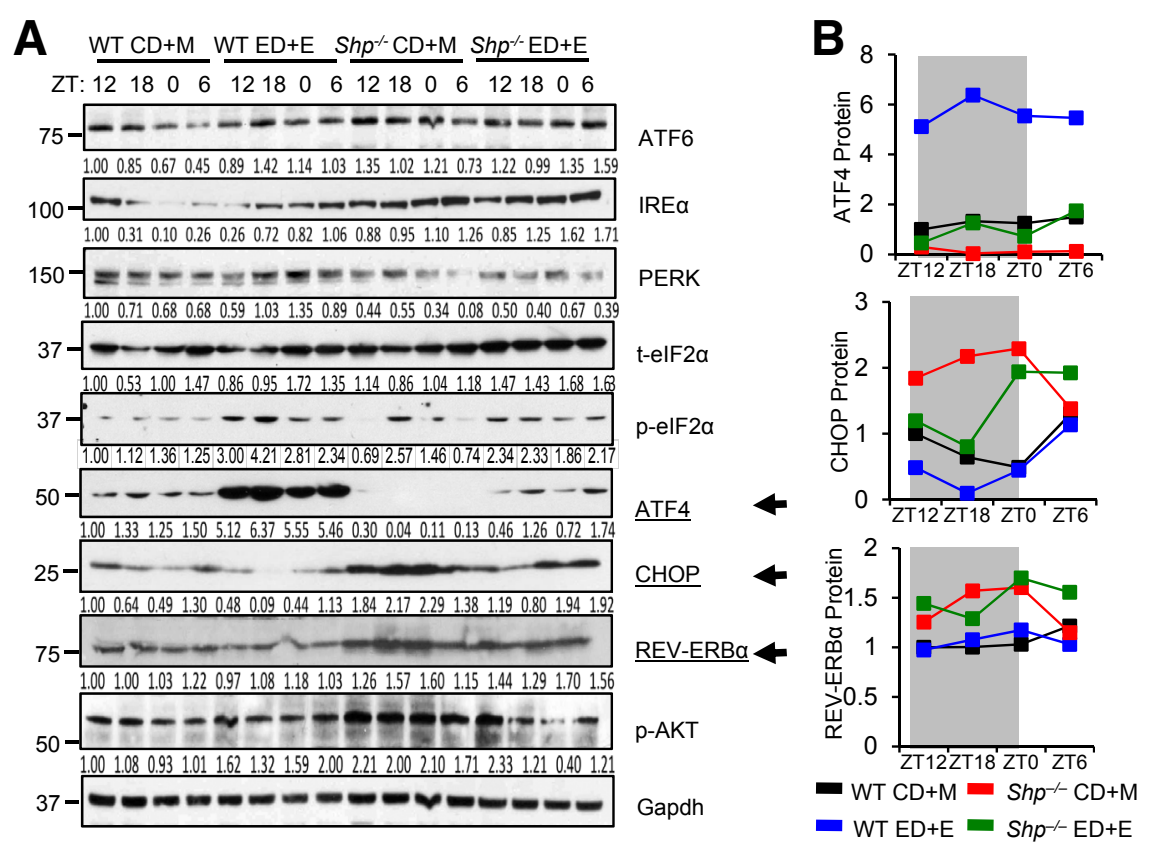

Figure 3 Shp deficiency disrupted maltoseand ethanol-mediated endoplasmic reticulum (ER) stress response. A: Western blot of hepatic proteins in the ER stress signaling pathways in WT and $\mathrm{Shp}^{-/-}$mice fed control diet + maltose binge $(C D+M)$ or ethanol diet + ethanol binge $(E D+E)$. Each lane represents equal amounts of protein pooled from five individual mice. Gray shading indicates dark cycle; white indicates light cycle. Arrows indicate most obviously changed proteins. Quantification of the intensity of each band was provided under each line and in panel B. ATF, activating transcription factor; CHOP, C/EBP homologous protein; Gapdh, glyceraldehyde-3phosphate dehydrogenase; IRE, inositol-requiring enzyme; p-eIF, phosphorylated eukaryotic translation initiation factor; PERK, PRKR-like endoplasmic reticulum kinase; $t$-eIF, total eukaryotic translation initiation factor; ZT, Zeitgeber time. induction in $\mathrm{WT}-\mathrm{ED}+\mathrm{E}$ versus $\mathrm{WT}-\mathrm{CD}+\mathrm{M}$, whereas in $\mathrm{Shp}^{-1-}$ mice ATF4 protein was largely impeded. Notably, despite that ATF4 protein is known as an activator of CHOP transcription, ${ }^{4}$ we observed opposite changes in the expression of CHOP protein. The CHOP protein was highly induced in $\mathrm{Shp}^{-1-}-\mathrm{CD}+\mathrm{M}$ versus $\mathrm{WT}-\mathrm{CD}+\mathrm{M}$ and was diminished in both WT and $S_{h} p^{-/-}$by ethanol $\left(\mathrm{Shp}^{-/-}\right.$$\mathrm{ED}+\mathrm{E}$ versus $S h p^{-1-}-\mathrm{CD}+\mathrm{M}$ and $\mathrm{WT}-\mathrm{ED}+\mathrm{E}$ versus WT$\mathrm{CD}+\mathrm{M}$ ) (Figure 3B). Although the inactivation of AKT by ER stress was reported to induce CHOP expression, ${ }^{24}$ we observed an induction in $\mathrm{p}-\mathrm{AKT}$ in $\mathrm{Shp}^{-1-} \mathrm{CD}+\mathrm{M}$ versus WT-CD + M. Most interestingly, REV-ERB $\alpha$ protein showed cyclic changes similar to the CHOP protein, which was highest in $\mathrm{Shp}^{-/-} \mathrm{CD}+\mathrm{M}$ but decreased in $S h p^{-/-}$-ED+E. Overall, there are two major observations. First, the distinct expression pattern of various ER stress markers suggests differential and unique responses of WT and $\mathrm{Shp}^{-1-}$ mice to the maltose and ethanol binge. Second, the results suggest a potential connection between CHOP and REV-ERB $\alpha$.

\section{The Expression of Hepatic CHOP and ATF4 Exhibited Different Alteration by Diet and Feeding Conditions in Mice}

The unexpected opposite expression pattern between CHOP and ATF4 protein in WT and Shp ${ }^{-/-}$mice observed above suggests a critical influence of maltose and ethanol feeding in combination with Shp deficiency. To further determine the relationship between CHOP and ATF4, we examined their expression levels using a different model (ie, in highfat and high-sucrose fed WT mice). Hepatic cleaved nSREBP-1c, REV-ERB $\alpha$, and CHOP proteins were similarly up-regulated by a high-fat and high-sucrose diet in both fed and fasted mice. In contrast, ATF4 protein was down-regulated by the high-fat and high-sucrose diet in both conditions (Figure 4). To confirm the previous report that ATF4 can activate Chop transcription directly, ${ }^{25}$ we overexpressed ATF4 and peroxisome proliferator-activated receptor $\gamma$, the known activators of Chop, in Hepal cells and observed the induction of Chop mRNA by both genes (Supplemental Figure S4A). In addition, tunicamycin (ER stress activator) induced both ATF4 and CHOP protein in MHC97H cells (Supplemental Figure S4B). These results suggest that, although ATF4 can function as a transcriptional activator of Chop, Chop expression in vivo is also tightly controlled by other transcription factors and nutrient status. As a result, Shp deficiency plus maltose binge appear to play a dominant role in increasing CHOP protein in $\mathrm{Shp}^{-1-} \mathrm{CD}+\mathrm{M}$, which completely masked the effect of ATF4, as seen in Figure 3.

\section{SHP Alters the Rhythmic Expression of CHOP via a REV-ERB $\alpha$-Dependent Mechanism}

REV-ERB $\alpha$ was reported being both a transcriptional activator $^{26}$ and repressor. ${ }^{27}$ The similar expression pattern between CHOP and REV-ERB $\alpha$ (Figure 3) raised an interesting question whether REV-ERB $\alpha$ acts as a transcription activator of CHOP. Because REV-ERB $\alpha$ can compete with ROR binding to the ROR element, ${ }^{28}$ we searched ROR element and identified it in mouse Chop promoter (Figure 5A). We cloned the native Chop promoter from -800 to 29 into a pGL3 vector for luciferase reporter assay. Overexpression of REV-ERB $\alpha$ significantly induced Chop promoter activity, which was inhibited by SHP coexpression and ethanol treatment 
A

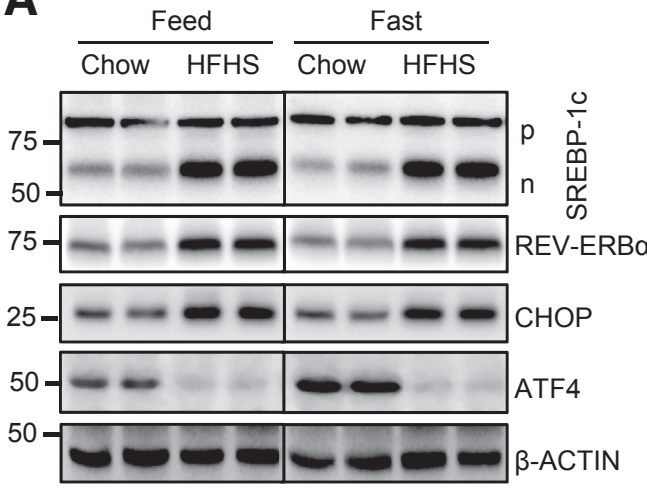

B
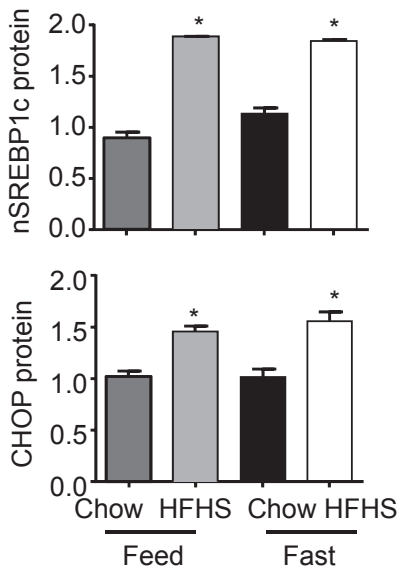
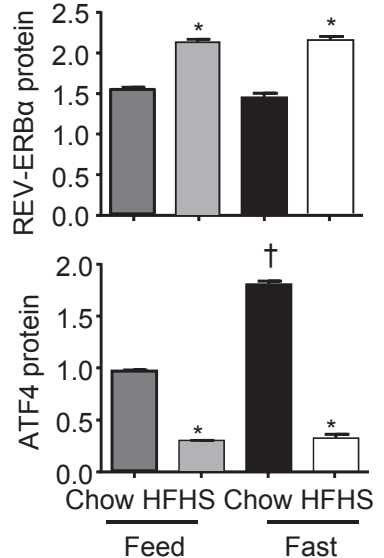

Figure 4 Hepatic C/EBP homologous protein (CHOP) and activating transcription factor (ATF4) expression was altered differentially by diets in mice. A: Western blot of hepatic sterol regulatory element-binding transcription factor 1c (SREBP-1C), REV-ERB $\alpha$, CHOP, and ATF4 protein in WT mice fed control chow or a high-fat and high-sucrose (HFHS) diet under feeding and fasting conditions. B: The quantification of protein levels of SREBP-1C, REV-ERB $\alpha$, CHOP, and ATF4. Samples were pooled and run in duplicate. Data are expressed as means \pm SD (B). $N=5$ individual mice per group (B). ${ }^{\star} P<0.05$, chow versus HFHS; ${ }^{\dagger} P<0.05$, feed chow versus fast chow. $n$, cleaved; $p$, precursor.

(Figure 5B). Interestingly, ROR $\alpha$ transactivated Chop promoter with less potency, but antagonized the activation of REV-ERB $\alpha$. The antagonism of ROR $\alpha$ to REV-ERB $\alpha$ activation was likely because of their competition for binding. Consistent with our previous observation, ${ }^{14}$ SHP did not affect ROR $\alpha$ activity. Mutation of ROR element in the Chop promoter greatly diminished $\mathrm{ROR} \alpha$ transactivation, and to a lesser extent, the REV-ERB $\alpha$ activity (Figure 5C). The results indicate that ROR element in the Chop promoter may have a stronger binding for REV-ERB $\alpha$ than for ROR $\alpha$, consistent with the higher potency of REV-ERB $\alpha$ to activate Chop promoter.

Chop mRNA was remarkably induced by both ROR $\alpha$ and REV-ERB $\alpha$ (Figure 5D). On the other hand, Shp repressed the effect of REV-ERB $\alpha$ but not ROR $\alpha$. In addition, CHOP protein was induced by REV-ERB $\alpha$ and its induction was
A

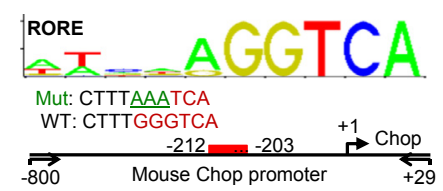

C Relative Chop Luc activity

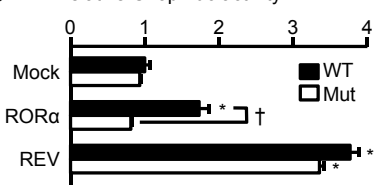

D

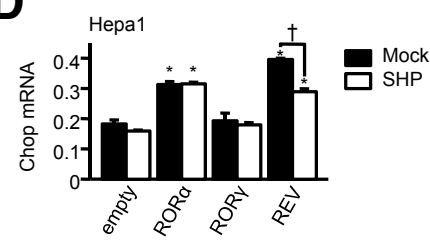

$\mathbf{F}$

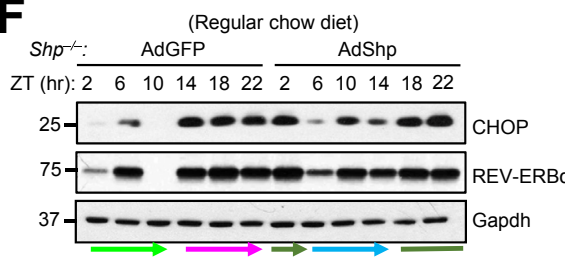

B

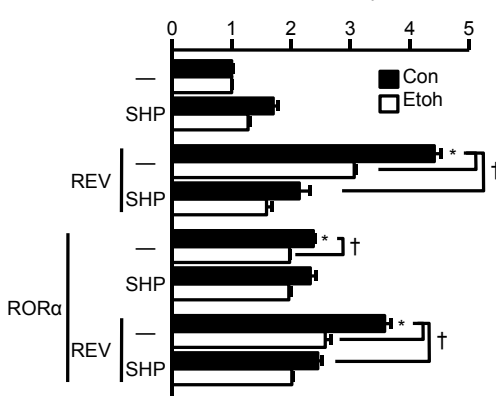

E
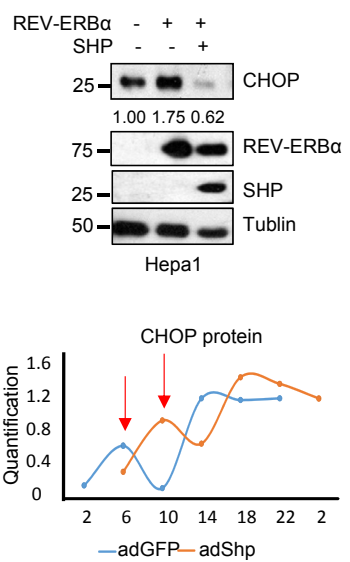

Figure 5 C/EBP homologous protein (Chop) gene transcription was activated by REV-ERB $\alpha$ and inhibited by SHP. A: Sequence logo of RAR-related orphan receptor (ROR) element (RORE; MA0071.1; JASPAR CORE Vertebrata database, http://jaspar. genereg.net) and RORE (red) in the mouse Chop promoter. Arrows indicate the location of primers for the construction of Chop promoter luciferase reporter. B: Luciferase reporter assay of Chop promoter activity in Hepa1 cells. The cells were transfected with mock (-), ROR $\alpha$, REV-ERB $\alpha(\operatorname{Rev})$, and SHP expression vectors, and treated with 0 (Con, gray) or $400 \mathrm{mmol} / \mathrm{L}$ (light blue) ethanol (Etoh) for 6 hours before the luciferase assay. C: Luciferase reporter assay of wild-type and mutant Chop promoter activity in Hepa1 cells. D: Quantitative PCR of Chop mRNA in ROR $\alpha-$, ROR $\gamma-$, or REVERB $\alpha$-overexpressing Hepa1 cells transfected with mock (black) or SHP (magenta) expression vectors. E: Western blot of proteins in Hepa1 cells transfected with expression plasmids of REV-ERB $\alpha$, SHP, or shRNA-REV-ERB $\alpha$. F: Western blot of hepatic CHOP and REV$\mathrm{ERB} \alpha$ in $\mathrm{Shp}^{-1-}$ mice reexpressed with adenoviral transduction of GFP (AdGFP) and SHP (AdSHP) on a regular chow diet (left panel) and the quantification of CHOP protein level (right panel). Livers were collected over a 12-hour/12-hour light/dark cycle. Colored arrows show the shift of the circadian pattern. Neon green arrow: peak on ZT6 in Shp ${ }^{-1-} /$ AdGFP; blue arrow: peak on ZT10 in $\mathrm{Shp}^{-/-}$/AdShp; magenta arrow: remain high expression from ZT14 to ZT22 in $\mathrm{Shp}^{-/-} /$AdGFP; green arrow and green line: remain high expression from ZT18 to ZT2 in Shp ${ }^{-/-} /$AdShp. Data are expressed as means \pm SD $(\mathbf{B}-\mathbf{D})$, or means $(\mathbf{F}) .{ }^{*} P<0.05$ versus empty vector; ${ }^{\dagger} P<0.05$. Con, control; luc, lucicerase; Mut, mutation; ZT, Zeitgeber time. 
abolished by SHP (Figure 5E). Thus, our results identified REV-ERB $\alpha$ as a new activator of CHOP.

Thus far, we observed that the maltose liquid diet, which is widely used as a control of ethanol diet in the alcohol research field, had a significant differential effect on TG metabolism in $\mathrm{Shp}^{-/-}$mice as compared to the regulator chow diet (Figure 1). To further determine the impact of SHP on the expression correlation between CHOP and REV-ERB $\alpha$, we overexpressed SHP using adenovirus in $S h p^{-1-}$ mice and collected samples over a 24-hour light/ dark cycle under the standard chow-fed condition. CHOP protein showed the same rhythmicity as REV-ERB $\alpha$ protein in control AdGFP mice (Figure 5F), which displayed a diurnal rhythm during the day (ZT: 2-6-10, low-high-low) with peak levels occurring constant during the night. Ectopic overexpression of SHP (AdSHP) disrupted the daily rhythm by causing a 4-hour shift in CHOP and REV-ERB $\alpha$ protein rhythmicity (ZT: 6-10-14: low-high-low) without altering the nocturnal rhythm. The quantification of $\mathrm{CHOP}$ protein level was shown on the right. Taken together, our results demonstrate that SHP alters the rhythmic expression of CHOP via a REV-ERB $\alpha$-dependent regulation.

\section{Knockdown REV-ERB $\alpha$ Prevented Ethanol-Induced Steatosis in Shp ${ }^{-/-}$Mice}

Next, we asked whether REV-ERB $\alpha$ played a physiological role in AFL in the presence (WT) or absence of Shp (Shp ${ }^{-1-}$ mice) by knockdown REV-ERB $\alpha$ in vivo (Supplemental Figure S5). We generated two shRNAs against REV-ERB $\alpha$ (shRev). The second shRev showed a strong effect in reducing SREBP-1c protein in Hepal cells (Supplemental Figure S6A), which was used in subsequent in vivo studies. ShRev down-regulated REV-ERB $\alpha$ protein and mRNA in liver, but not in WT mouse brain (Supplemental Figure S6, $\mathrm{B}-\mathrm{D})$, suggesting a liver-specific effect. Consistent with the
A

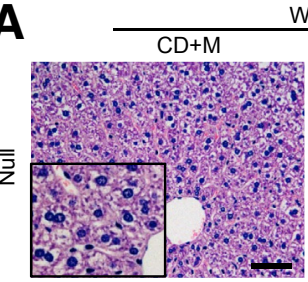

WT
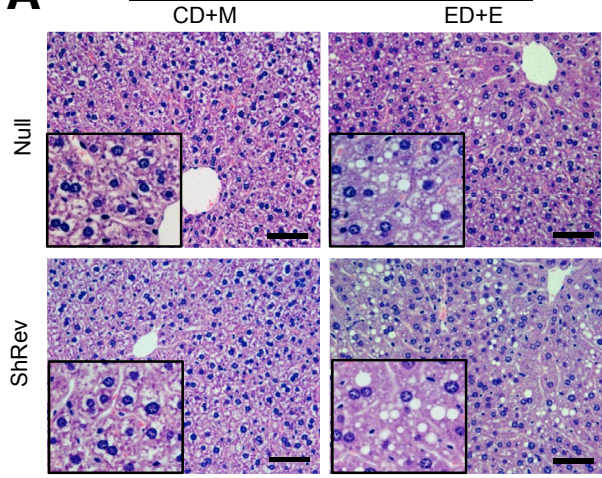

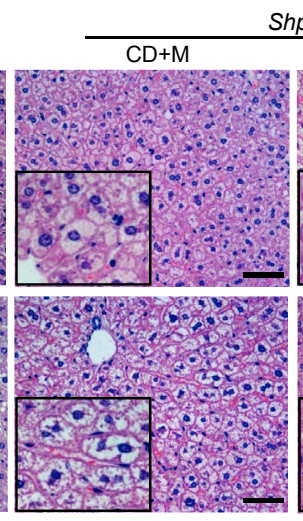

Shp-/

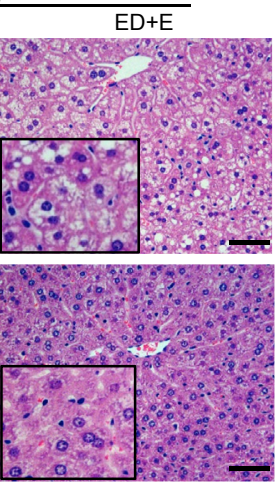

B

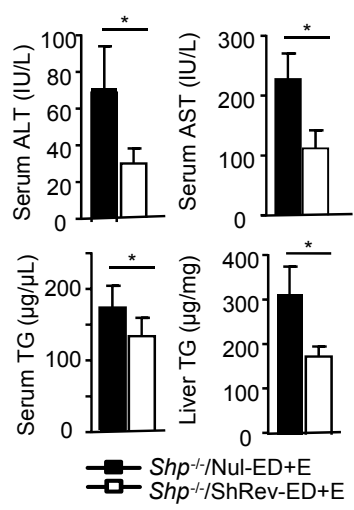

C Shp $^{-/ /} /$Null-ED+E
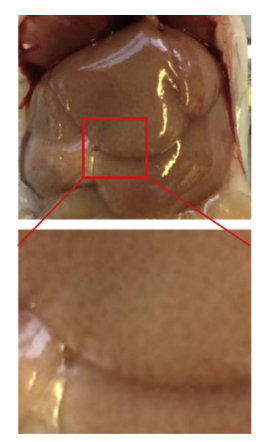

$\mathrm{Shp}^{-/ /}$ShRev-ED+E
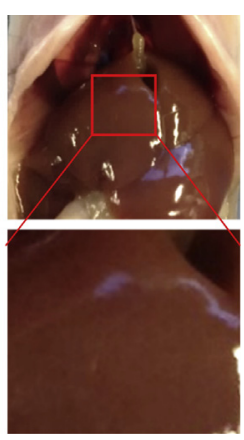

$\mathrm{Sh}^{-/-/ N u l l-E D+E} \quad \mathrm{Shp}^{-/ /}$ShRev-ED+E

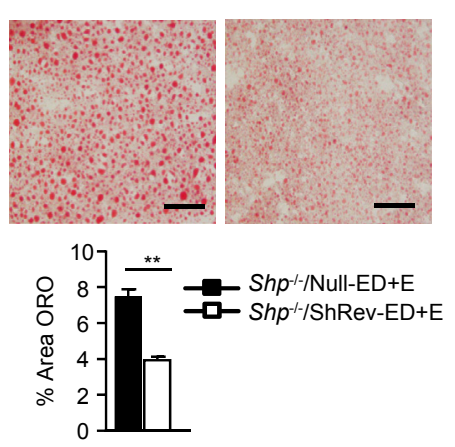

D

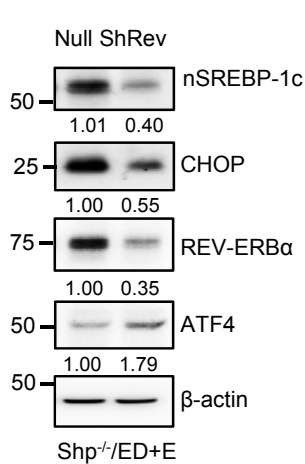

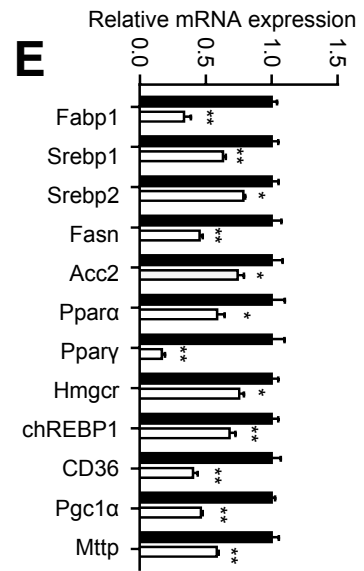

Figure 6 Knockdown Rev-Erba impeded ethanol-induced steatosis in Shp ${ }^{-/-}$mice. A: Hematoxylin and eosin staining of liver sections in WT and Shp ${ }^{-/-}$ mice with Rev-Erb $\alpha$ knockdown and then fed control diet + maltose binge (CD+M) or ethanol diet + ethanol binge (ED+E). Sampling at Zeitgeber time (ZT) 12. Null: control vector for Sh-Rev-Erb $\alpha$. B: Serum alanine transaminase (ALT), aspartate transaminase (AST), triglyceride (TG), and liver TG in Shp ${ }^{-/-} /$Null and $^{-1}$ $\mathrm{Shp}^{-/-} / \mathrm{Sh}$-Rev mice fed with ED+E. C: Gross morphology (left panels) and oil red 0 staining of liver sections (right panels) in Shp ${ }^{-/-} / \mathrm{Null}^{-}$and Shp ${ }^{-/-} / \mathrm{Sh}_{-}$ Rev mice fed with ED+E. D: Western blot of liver protein in $S h p^{-1-} /$ Null and Shp ${ }^{-1-} /$ Sh-Rev mice fed ED+E. E: Quantitative PCR of hepatic lipogenic gene mRNA in Shp ${ }^{-/} /$Null and Shp ${ }^{-/-} /$Sh-Rev mice fed ED+E. Data are expressed as means \pm SD (B, C, and E). ${ }^{*} P<0.05,{ }^{* *} P<0.01$ versus Shp ${ }^{-/} /$Null-ED + E. Scale bar $=20 \mu \mathrm{m}$ (A and C). Original magnifications: $\times 20$ (A, main images); $\times 40$ (A, insets). Acc, acetyl-CoA carboxylase; ATF, activating transcription factor; CD36, cluster of differentiation 36; CHOP, C/EBP homologous protein; chREBP, carbohydrate-responsive element-binding protein; Fabp, fatty acidbinding protein; Fasn, fatty acid synthase; Hmgcr, 3-hydroxy-3-methylglutaryl-CoA reductase; Mttp, microsomal triglyceride transfer protein; Pgc1 $\alpha$, peroxisome proliferator-activated receptor $\gamma$ coactivator 1- $\alpha$; Ppar, peroxisome proliferator-activated receptor; Srebp, sterol regulatory element-binding transcription factor. 
results presented in Figure 3, ATF4 protein was highly induced by ethanol binge in WT/null mice (null: shRev control), whereas it was not affected by shRev (Supplemental Figure S6E). Rev-Erb $\alpha$ mRNA was also down-regulated by shRev in $\mathrm{Shp}^{-1-}$ mice (Supplemental Figure S6F).

Histology analysis by hematoxylin and eosin staining revealed several interesting observations. In WT mice, shRev-ED+E moderately increased steatosis compared with null-ED+E (Figure 6A). However, in $S h p^{-1-}$ mice, the steatosis (more in the form of microsteatosis) in shRev-CD $+\mathrm{M}$ was somewhat increased compared with null-CD + M. However, these differences did not reach statistical significance, as determined by liver TG content (Supplemental Figure S7). The steatosis induced by ethanol binge in $S h p^{-1-}$ /null-ED+E liver was largely prevented in $S h p^{-/-} /$shRev-ED+E liver, which was accompanied by the decrease in serum ALT, serum AST, serum TG, and liver TG in $S h p^{-1-} / \mathrm{shRev-ED+E}$ versus $S h p^{-/-} /$null-ED $+\mathrm{E}$ (Figure 6B). We also observed alleviated pale liver color and markedly reduced neutral lipid staining in $\mathrm{Shp}^{-1-}$ shRev-ED+E versus $S h p^{-1-} /$ null-ED + E (Figure 6C). The nSREBP-1c and CHOP protein (Figure 6D) and many genes involved in lipid metabolism (Figure 6E) were down-regulated by shRev. Therefore, Shp and Rev-Erb $\alpha$ double deficiency prevented ethanol-induced alcoholic fatty liver.

\section{Discussion}

Fatty liver (steatosis) is the collection inside hepatocytes of excessive amounts of fat, which consists mainly of TG. An important cause of fatty liver is alcohol abuse, referred to as AFL. Macrovesicular alcoholic steatosis is a common form of AFL and is featured by the presence of large sharp fat droplets in hepatocytes, and the nuclei are in a peripheral location. ${ }^{29}$ Although alcohol impairs circadian rhythm in liver, ${ }^{30}$ the influence of nuclear receptor circadian regulators on alcoholic fatty liver remains unclear. Our studies identified two new players (ie, nuclear receptors SHP and REV-ERB $\alpha$ ) that are important in mediating the development of AFL (Figure 7).

The most widely used model for alcoholic liver injury is feeding with the Lieber-DeCarli diet containing ethanol for 4 to 6 weeks $^{31}$; however, this model only induces mild steatosis and slight elevation of serum ALT with low or no
A

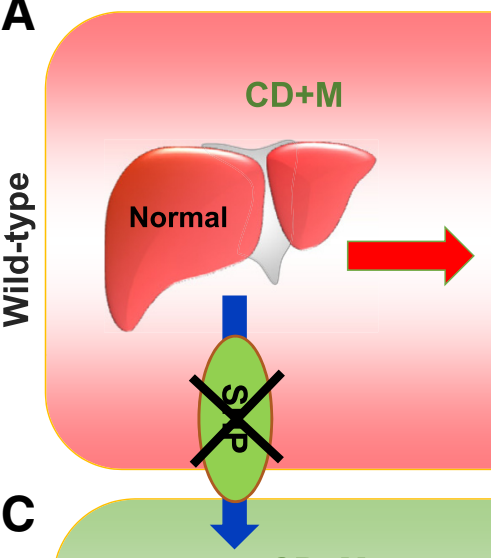

$\mathrm{CD}+\mathrm{M}$

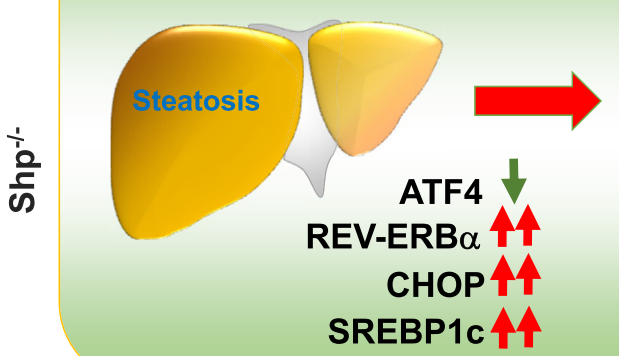

$E D+E$

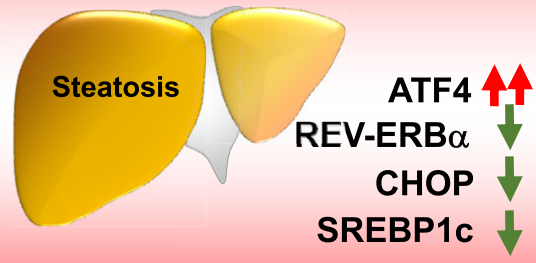

$E D+E$

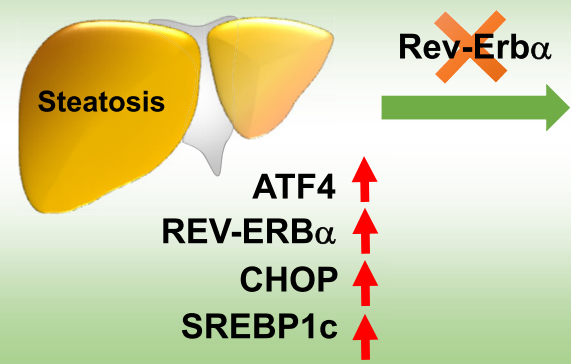

B
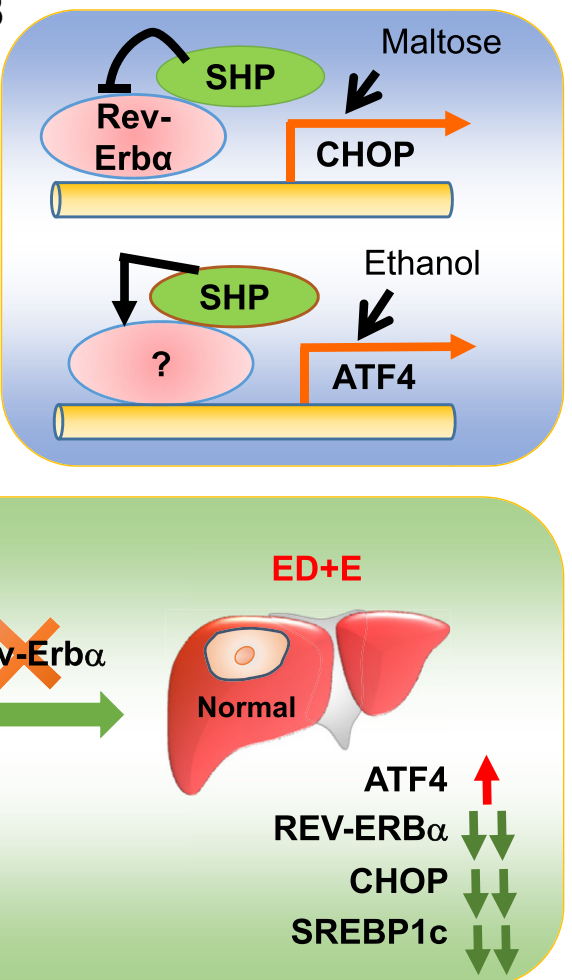

Figure 7 Schematic summarizing major findings in this study. A: WT mice: ethanol diet + ethanol binge (ED+E) induces steatosis, which correlates with a marked up-regulation of activating transcription factor 4 (ATF4) protein but down-regulation of C/EBP homologous protein (CHOP) and sterol regulatory element-binding transcription factor 1c (SREBP-1c) protein. However, knockdown ATF4 does not prevent steatosis induced by ED+E. B: Top image: SHP inhibits Chop transcriptional activation by REV-ERB $\alpha$. Chop induction by loss of SHP repression is augmented by control diet + maltose binge (CD $+M)$. Bottom image: SHP activates ATF4 via an unknown mechanism. ED+E induces ATF4 while transiently down-regulating SHP expression. C: $\mathrm{Shp}^{-/-}$mice: $\mathrm{CD}+\mathrm{M}$ causes microvesicular steatosis, which correlates with a sharp elevation of CHOP, SREBP-1C, and REV-ERB $\alpha$ protein but a diminished ATF4 protein. On the contrary, $\mathrm{ED}+\mathrm{E}$-induced steatosis correlates with moderate up-regulation of ATF4, CHOP, SREBP-1C, and REV-ERB $\alpha$ protein relative to WT-ED+E. Knockdown REV-ERB $\alpha$ prevents $\mathrm{ED}+\mathrm{E}$-induced steatosis. 
liver inflammation. ${ }^{15}$ The Gao-binge model is a relatively new model, which represents an acute model of alcoholinduced liver injury and has been widely used by other research groups. ${ }^{18,19}$ One limitation done by other groups is that samples are collected at one time point 9 hours after binge and the liver phenotype (inflammation and lipid accumulation) may represent transient responses to acute injury. A novel aspect of our study is to examine lipid metabolic changes by collecting samples over a 24-hour light/dark cycle. Another novelty of our study is to determine the impact of nuclear receptors SHP and REV-ERB $\alpha$ in AFL, both of which are important regulators of the circadian clock machinery in the liver.

The function of SHP has been well characterized by us and others in cholesterol and lipid metabolism associated with NAFLD. ${ }^{11,14,32}$ However, SHP's role in ALD remains largely unknown. Therefore, the studies were conducted using both WT and $S h p^{-/-}$mice. Phenotypically, several unexpected findings were observed. The serum and hepatic lipid profile, as well as the expression of lipid synthetic genes and ER stress markers, exhibit strong but distinct cyclic variations in WT and $S h p^{-/-}$mice. ED+E induces steatosis in WT mice, which correlates with a marked upregulation of ATF4 protein; it leads to a down-regulation of CHOP and SREBP-1c protein. On the contrary, CD+M seems to cause a variant of steatosis (namely, microvesicular steatosis) in $S h p^{-/-}$mice, which is accompanied by a sharp elevation of CHOP, SREBP-1c, and REV-ERB $\alpha$ protein but a diminished ATF4 protein. The differential activation of ATF4 and CHOP, which corresponds to steatosis in WT mice by ethanol and microvesicular steatosis in $\mathrm{Shp}^{-/-}$mice by maltose, respectively, suggest a divergent function of both proteins to potentially regulate two different forms of steatosis. Unfortunately, knockdown ATF4 (Supplemental Figure S8) did not diminish ED+E-induced steatosis in WT mice (data not shown), suggesting its activation by ethanol is likely the consequence but not the cause of steatosis. One possibility is that $\mathrm{ED}+\mathrm{E}$ may cause mitochondria damage and decrease mitochondria $\beta$ oxidation. ${ }^{1}$ The microvesicular fat deposition in the short timelines of these studies may reflect an intermediate stage of lipid formation. Nonetheless, because liver transplant donors with macrosteatosis (common) and microsteatosis (less common) respond differently to intermittent hepatic inflow occlusion during tumor hepatectomy, ${ }^{33}$ our results may contribute to a better understanding of the molecular basis of microsteatosis.

REV-ERB $\alpha$ is an integrator of circadian rhythms and metabolism in the liver. ${ }^{34}$ Dual depletion of Rev-Erb $\alpha$ and Rev-Erb $\beta$ disrupts lipid homeostasis gene networks, ${ }^{35}$ and single deletion of Rev-Erb $\alpha$ leads to modest hepatosteatosis. ${ }^{36}$ However, these early studies were performed in NAFLD and no detailed studies have been done to elucidate the function of REV-ERB $\alpha$ in AFL. Interestingly, REV-ERB $\alpha$ interacts with SHP and exerts as a transcriptional repressor of NPAS2 expression in NAFLD. ${ }^{14}$ In this study, we demonstrate that REV-ERB $\alpha$ acts as a transcriptional activator of CHOP expression and contributes to the development of AFL under Shp-deficient conditions. Therefore, REV-ERB $\alpha$ appears to have bidirectional transcriptional effects as a repressor or an activator, depending on the gene promoter as well as the context of lipid metabolic dysregulation (NAFLD versus AFL). Of critical importance, REV-ERB $\alpha$ knockdown prevents ethanol diet plus ethanol binge-induced steatosis in $\mathrm{Shp}^{-/-}$mice. Interestingly, knockdown of REV-ERB $\alpha$ shows minimal effect in altering lipid profile in WT mice fed with maltose or ethanol, signifying the importance of REV-ERB $\alpha$ and SHP cross talk in the control of ALD. ${ }^{37}$

In conclusion, our study elucidates a crucial cross talk between two circadian clock modulators, SHP and REV$\mathrm{ERB} \alpha$, to regulate AFL by the integration of ER stress marker CHOP. SHP inhibits CHOP activation by REV$\mathrm{ERB} \alpha$; thus, the permanent loss of SHP repression in $S h p^{-/-}$mice enhances CHOP induction by maltose, which may contribute to the development of steatosis. In addition, double deficiency of $S h p$ and Rev-Erb $\alpha$ prevented mice from developing ethanol-induced steatosis. Because most researchers in the alcohol field study ALD using samples collected from a single time point, our study represents an important shift in alcohol research by encompassing the liver clock component. The knowledge gained from this study is of clinical importance when developing therapeutic agents to target SHP and REV-ERB $\alpha$ in NAFLD versus AFL.

\section{Acknowledgments}

Z.Y. and H.T. performed experiments and prepared the manuscript; Y.Z., S.L., C.H., Y.H., and G.M.V. performed experiments; L.W. supervised the work and wrote the manuscript.

\section{Supplemental Data}

Supplemental material for this article can be found at http://dx.doi.org/10.1016/j.ajpath.2016.07.014.

\section{References}

1. Williams JA, Manley S, Ding WX: New advances in molecular mechanisms and emerging therapeutic targets in alcoholic liver diseases. World J Gastroenterol 2014, 20:12908-12933

2. You M, Jogasuria A, Lee K, Wu J, Zhang Y, Lee YK, Taylor C, Sadana P: Signal transduction mechanisms of alcoholic fatty liver disease: emerging role of lipin-1. Curr Mol Pharmacol 2015, [Epub ahead of print] doi:10.2174/1874467208666150817112109

3. Ji C: Mechanisms of alcohol-induced endoplasmic reticulum stress and organ injuries. Biochem Res Int 2012, 2012:216450

4. Malhi H, Kaufman RJ: Endoplasmic reticulum stress in liver disease. J Hepatol 2011, 54:795-809

5. Tsuchiya H, da Costa KA, Lee S, Renga B, Jaeschke H, Yang Z, Orena SJ, Goedken MJ, Zhang Y, Kong B, Lebofsky M, Rudraiah S, 
Smalling R, Guo G, Fiorucci S, Zeisel SH, Wang L: Interactions between nuclear receptor SHP and FOXA1 maintain oscillatory homocysteine homeostasis in mice. Gastroenterology 2015, 148:1012-1023.e14

6. Rudraiah S, Zhang X, Wang L: Nuclear receptors as therapeutic targets in liver disease: are we there yet? Annu Rev Pharmacol Toxicol 2016, $56: 605-626$

7. Zhang $\mathrm{Y}$, Hagedorn $\mathrm{CH}$, Wang L: Role of nuclear receptor SHP in metabolism and cancer. Biochim Biophys Acta 2011, 1812: 893-908

8. Zhou T, Zhang Y, Macchiarulo A, Yang Z, Cellanetti M, Coto E, Xu P, Pellicciari R, Wang L: Novel polymorphisms of nuclear receptor SHP associated with functional and structural changes. J Biol Chem 2010, 285:24871-24881

9. Wang L, Lee YK, Bundman D, Han Y, Thevananther S, Kim CS, Chua SS, Wei P, Heyman RA, Karin M, Moore DD: Redundant pathways for negative feedback regulation of bile acid production. Dev Cell 2002, 2:721-731

10. Zhang Y, Xu N, Xu J, Kong B, Copple B, Guo GL, Wang L: E2F1 is a novel fibrogenic gene that regulates cholestatic liver fibrosis through the Egr-1/SHP/EID1 network. Hepatology 2014, 60:919-930

11. Huang J, Iqbal J, Saha PK, Liu J, Chan L, Hussain MM, Moore DD, Wang L: Molecular characterization of the role of orphan receptor small heterodimer partner in development of fatty liver. Hepatology 2007, 46:147-157

12. Pan X, Zhang $\mathrm{Y}$, Wang L, Hussain MM: Diurnal regulation of MTP and plasma triglyceride by CLOCK is mediated by SHP. Cell Metab 2010, 12:174-186

13. Wang L, Liu J, Saha P, Huang J, Chan L, Spiegelman B, Moore DD: The orphan nuclear receptor SHP regulates PGC-1alpha expression and energy production in brown adipocytes. Cell Metab 2005, 2: $227-238$

14. Lee SM, Zhang Y, Tsuchiya H, Smalling R, Jetten AM, Wang L: Small heterodimer partner/neuronal PAS domain protein 2 axis regulates the oscillation of liver lipid metabolism. Hepatology 2015, 61: 497-505

15. Bertola A, Mathews S, Ki SH, Wang H, Gao B: Mouse model of chronic and binge ethanol feeding (the NIAAA model). Nat Protoc 2013, 8:627-637

16. Zhang Y, Liu C, Barbier O, Smalling R, Tsuchiya H, Lee S, Delker D, Zou A, Hagedorn CH, Wang L: Bcl2 is a critical regulator of bile acid homeostasis by dictating Shp and IncRNA H19 function. Sci Rep 2016, 6:20559

17. Yang Z, Zhang Y, Wang L: A feedback inhibition between miRNA127 and TGFbeta/c-Jun cascade in HCC cell migration via MMP13. PLoS One 2013, 8:e65256

18. Chen P, Miyamoto Y, Mazagova M, Lee KC, Eckmann L, Schnabl B: Microbiota protects mice against acute alcohol-induced liver injury, alcoholism. Clin Exp Res 2015, 39:2313-2323

19. Roh YS, Zhang B, Loomba R, Seki E: TLR2 and TLR9 contribute to alcohol-mediated liver injury through induction of CXCL1 and neutrophil infiltration. Am J Physiol Gastrointest Liver Physiol 2015, 309:G30-G41

20. Watanabe M, Houten SM, Wang L, Moschetta A, Mangelsdorf DJ, Heyman RA, Moore DD, Auwerx J: Bile acids lower triglyceride levels via a pathway involving FXR, SHP, and SREBP-1c. J Clin Invest 2004, 113:1408-1418
21. Ferre P, Foufelle F: Hepatic steatosis: a role for de novo lipogenesis and the transcription factor SREBP-1c, diabetes. Obesity Metab 2010, 12(Suppl 2):83-92

22. Purohit V, Gao B, Song BJ: Molecular mechanisms of alcoholic fatty liver, alcoholism. Clin Exp Res 2009, 33:191-205

23. Ji C: New insights into the pathogenesis of alcohol-induced ER stress and liver diseases. Int J Hepatol 2014, 2014:513787

24. Hyoda K, Hosoi T, Horie N, Okuma Y, Ozawa K, Nomura Y: PI3KAkt inactivation induced CHOP expression in endoplasmic reticulumstressed cells. Biochem Biophys Res Commun 2006, 340:286-290

25. Averous J, Bruhat A, Jousse C, Carraro V, Thiel G, Fafournoux P: Induction of $\mathrm{CHOP}$ expression by amino acid limitation requires both ATF4 expression and ATF2 phosphorylation. J Biol Chem 2004, 279: $5288-5297$

26. Harding HP, Lazar MA: The orphan receptor Rev-ErbA alpha activates transcription via a novel response element. Mol Cell Biol 1993, 13: 3113-3121

27. Wang J, Yin L, Lazar MA: The orphan nuclear receptor Rev-erb alpha regulates circadian expression of plasminogen activator inhibitor type 1. J Biol Chem 2006, 281:33842-33848

28. Guillaumond F, Dardente H, Giguere V, Cermakian N: Differential control of Bmall circadian transcription by REV-ERB and ROR nuclear receptors. J Biol Rhythms 2005, 20:391-403

29. Yerian L: Histopathological evaluation of fatty and alcoholic liver diseases. J Dig Dis 2011, 12:17-24

30. Udoh US, Valcin JA, Gamble KL, Bailey SM: The molecular circadian clock and alcohol-induced liver injury. Biomolecules 2015, 5: 2504-2537

31. Everitt H, Hu M, Ajmo JM, Rogers CQ, Liang X, Zhang R, Yin H, Choi A, Bennett ES, You M: Ethanol administration exacerbates the abnormalities in hepatic lipid oxidation in genetically obese mice. Am J Physiol Gastrointest Liver Physiol 2013, 304:G38-G47

32. Datta S, Wang L, Moore DD, Osborne TF: Regulation of 3-hydroxy-3methylglutaryl coenzyme A reductase promoter by nuclear receptors liver receptor homologue-1 and small heterodimer partner: a mechanism for differential regulation of cholesterol synthesis and uptake. $\mathrm{J}$ Biol Chem 2006, 281:807-812

33. Han S, Kim G, Lee SK, Kwon CH, Gwak M, Lee S, Ha S, Park CK, Ko JS, Joh J: Comparison of the tolerance of hepatic ischemia/reperfusion injury in living donors: macrosteatosis versus microsteatosis. Liver Transpl 2014, 20:775-783

34. Duez H, Staels B: Rev-erb-alpha: an integrator of circadian rhythms and metabolism. J Appl Physiol 2009, 107:1972-1980

35. Cho H, Zhao X, Hatori M, Yu RT, Barish GD, Lam MT, Chong LW, DiTacchio L, Atkins AR, Glass CK, Liddle C, Auwerx J, Downes M, Panda S, Evans RM: Regulation of circadian behaviour and metabolism by REV-ERB-alpha and REV-ERB-beta. Nature 2012, 485:123-127

36. Bugge A, Feng D, Everett LJ, Briggs ER, Mullican SE, Wang F, Jager J, Lazar MA: Rev-erbalpha and Rev-erbbeta coordinately protect the circadian clock and normal metabolic function. Genes Dev 2012, 26:657-667

37. Duez H, van der Veen JN, Duhem C, Pourcet B, Touvier T, Fontaine C, Derudas B, Bauge E, Havinga R, Bloks VW, Wolters H, van der Sluijs FH, Vennstrom B, Kuipers F, Staels B: Regulation of bile acid synthesis by the nuclear receptor Rev-erbalpha. Gastroenterology 2008, 135:689-698 